\title{
Conf $-940365--1$
}

Engineering Physics and Mathematics Division

Task Automation in a Successful Industrial Telerobot*

P. F. Spelt

Oak Ridge National Laboratory

and

S. L. Jones

Remotec, Inc.

Oak Ridge, TN 37830

\section{DISCLAIMER}

\begin{abstract}
This report was prepared as an account of work sponsored by an agency of the United States Government. Neither the United States Government nor any agency thereof, nor any of their employees, makes any warranty, express or implied, or assumes any legal liability or responsibility for the accuracy, completeness, or usefulness of any information, apparatus, product, or process disclosed, or represents that its use would not infringe privately owned rights. Reference herein to any specific commercial product, process, or service by trade name, trademark, manufacturer, or otherwise does not necessarily constitute or imply its endorsement, recommendation, or favoring by the United States Government or any agency thereof. The views and opinions of authors expressed herein do not necessarily state or reflect those of the United States Government or any agency thereof.
\end{abstract}

Submitted to AIAA/NASA Conference on Intelligent Robots in Field, Factory, Service, and Space (CIRFFSS '94), Houston, TX, March 21-24, 1994

* Research sponsored by the Office of Nuclear Energy, Office of Technology Support Programs, U.S. Department of Energy, under contract No. DE-AC05-84OR21400 with Martin Marietta Energy Systems, Inc. 


\title{
Task Automation in a Successful Industrlal Telerobot"
}

\author{
Philip F. Spelt \\ Center for Engineering Systems Advanced Research \\ Oak Ridge National L.aboratory \\ P. O. Box 2008 \\ Oak Ridge, Tennessee 37831-6364
}

and

Sammy L. Jones

Remotec, Inc.

114 Union Valley Road

Oak Ridge, Tennessee 37830

\begin{abstract}
In this paper, we discuss cooperative work by Oak Ridge National Laboratory and Remotec ${ }^{\circledR}$, Inc., to automate components of the operator's workload using Remotec's Andros telerobot, thereby providing an enhanced user interface which can be retrofit to existing fielded units as well as being incorporated into new production units. Remotec's Andros robots are presently used by numerous electric utilities to perform tasks in reactors where substantial exposure to radiation exists, as well as by the armed forces and numerous law enforcement agencies. The automation of task components, as well as the video graphics display of the robot's position in the environment, will enhance all tasks performed by these users, as well as enabling performance in terrain where the robots cannot presently perform due to lack of knowledge about, for instance, the degree of tilt of the robot. Enhanced performance of a successful industrial mobile robot leads to increased safety and efficiency of performance in hazardous erivironments. The addition of these capabilities will greatly enhance the utility of the robot, as well as its marketability.
\end{abstract}

\section{Introduction}

The robotic system described in this paper results from a cooperative effort by the Center for Engineering Systems Advanced Research (CESAR), at Oak Ridge National Laboratory (ORNL), and Remotec ${ }^{\circledR}$, Inc., a company located in Oak Ridge, TN. CESAR, sponsored by the Engineering Sciences Program of the Department of Energy (DOE) Office of Basic Energy Sciences, represents a core long-term basic research program in intelligent machines. CESAR research includes studies in multiple cooperating robots, multisensor data analysis and fusion, control of mobile robots and manipulators, machine learning, and embedded high performance computing. With support from the DOE Office of Nuclear Energy, CESAR has been performing applied robotics research, systems integration, and has provided overall coordination and management of a

"Research supported by the Office of Nuclear Energy, Office of Technology Support Programs, U.S. Department of Energy, under contract No., DE-ACC5-840R21400 with Martin Marietta Energy Systems, inc. consortium of four university research groups (Florida, Michigan, Tennessee, Texas) in a program aimed at robotics for advanced nuclear power stations and other hazardous environments.

Remotec is a world leader in research and development of remote robotic technology for hazardous operation in nuclear plants, police/military explosive ordnance disposal, and fire fighting. The company's family of robots have found a worldwide clientele. They are being used by several nuclear utility industries and national research laboratories to perform waste handling, surveillance, and surveying. This paper describes the addition of a system of sensors, encoders and the required computing power to integrate the information gleaned from these sensors to enhance the teleoperation of a successful industrial mobile robot. All hardware additions are performed in a manner which preserves the factory-designed resistance of the chassis to environmental contamination. Moreover, as will be described in detail below, the functional additions which enhance the teleoperation of this robot are done in a manner which preserves the original factory functionality. This is desirable because the retrofitting of an enhanced interface to existing robots should require as little additional training of already skilled operators as possible.

\section{The Andros Mk VIRobot}

The mobile platform of the ANDROS robot, shown in Figure 1, consists of six cleated tracks including a pair of main driving tracks. Separate motors to drive two pairs of auxiliary tracks: a pair of articulated front tracks, and an additional pair of articulated rear tracks. This unique design enables the robot to climb stairs and slopes, crawl over obstacles and ditches, make turns in tight spaces, raise the entire robot body, and maneuver over rough terrain with different surface conditions. The ANDROS manipulator arm has five degrees-of-freedom (DOF), with a 210 degree pivot range for both shoulder and elbow. An additional DOF is provided by a torso rotation joint, in addition to the platform mobility. This configuration allows the arm to occupy a minimum space for its home position while This paper is declared a work of the U.S. Government and is not subject to copyright protection in the United States. 


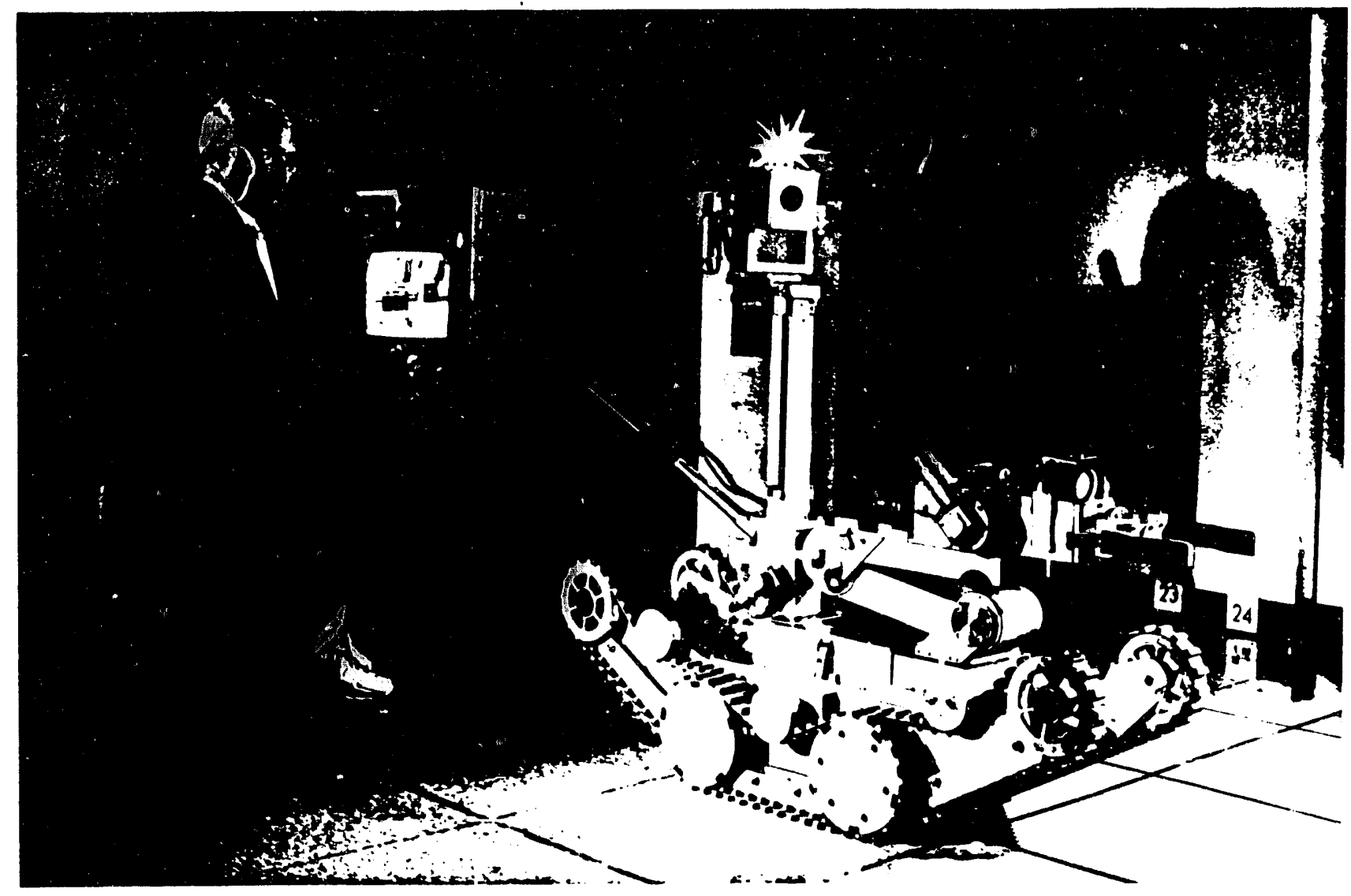

Figure 1. Andros MK VI telerobot with control console in background.

providing maximum reach by folding down and extending straight out, respectively. Each joint is manually controlled with variable speed by individual switches on the control station. The wrist has pitch and six-inch extension capability, as well as continuous rotation, and the gripper has two parallel fingers controlled by servo-motors. The maximum lifting capacity is $40 \mathrm{~kg}$.

The control station, shown in the background of Figure 1 . consists of a switch pad with all the switches required to operate the ANDROS robot; a control console with a color television monitor, speaker, and microphone; and a console cable reel with a manual brake and handcrank for the 100-m tether. Two video cameras are mounted aboard the chassis: a monochrome fixed-focus camera with automatic aperture is attached to the arm, and serves as a navigation camera when the arm is parked in the home position; there is also a color camera mounted on an extendible tower with pan, tilt, zoom, and focus capabilities under operator control. This camera serves as a general surveillance camera for both navigation and manipulator arm tasks.

In addition to the two-camera video feedback from the robot, two-way audio communication is available through a microphone/speaker system aboard the chassis and on the console. All told, there are 24 control functions on the control panel of the console, including the talk and volume switches for audio communication. Manipulating these control devices to smoothly control the robot and accomplish a task in the workplace requires considerable skill and practice on the part of the operator. In situations where the robot is out of direct sight of the operator, work must halt while the two cameras are used to assess current robot pose and the surrounding environment.

\section{Workload considerations}

Excessive workload on an operator of such a telerobot can degrade or slow down performance due to the number of task components which are manually performed. These components include manipulation of the cameras to monitor robot pose and tether placement, as well as to observe the effects of remote actions on the surrounding environment. In many cases, task pertormance must be iriterrupted to permit the operator to observe changes in robot pose as work progresses. The capacity to provide sensor feedback to the operator aboui robot position, articulator and arm position, and proximity of obstacles in the immediate environment. would greatly enhance overall performance of the system. In addition, automation of task components requires sensory feedback from the environment as well as encoder feedback about the positions of various robot components.

The procedure of automating a telerobot requires the addition of computer pcwer to the robot, along with a variety of sensors and encoders to provide information about the robot's performance in and relationship to its environment. Custom software is required to integrate the encoder and sensor information and to use this information to provide automated control input to the 


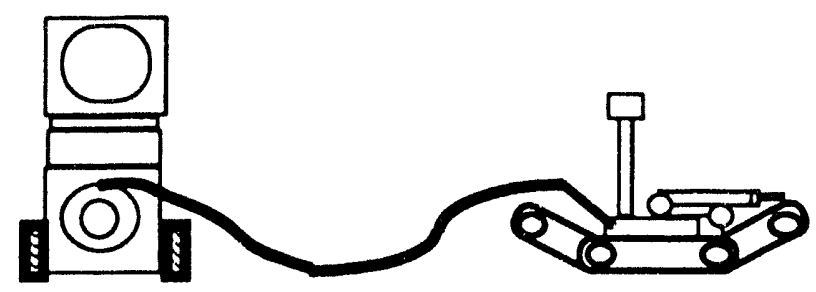

Fig. 2a. Factory configuration of Andros Mk VI robot and control console

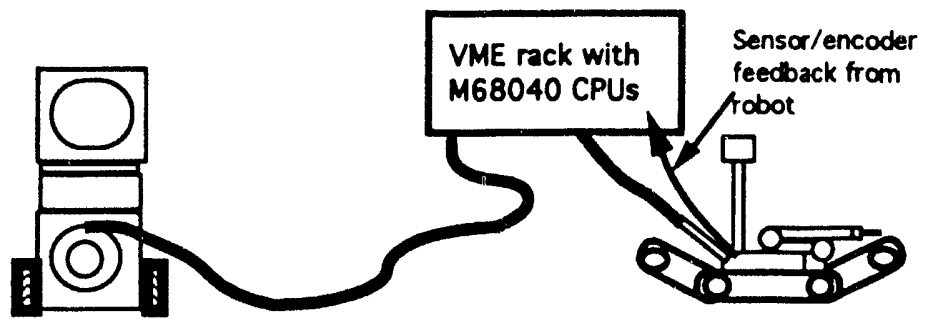

Fig. 2b. Additional computing power added to Andros MK VI robot and control console

Figure 2. Illustration of original and enhanced Andros robot configuration.

robot. To be most effective, a variety of tasks must be automated, including obstacle detection and avoidance, planned manipulations by the arm and end-effector, and eye-gaze control of video camera pan and tilt. Addition of these capabilities will greatly enhance the teleoperation of an already successful industrial mobile robot. In order to accomplish these enhancements, a cooperative research and development agreement (CRADA) has been implemented between Remotec and ORNL. This CRADA involves equal inputs of time, effort and money on the part of both parties in order to create the enhanced robot described.

\section{Enhancements to the Andros robol}

As described above, the enhancements to the Andros robot require the addition of environmental sensors, encoders for the various robot movable parts, and computing power to provide the intelligence to integrate sensor and encoder information and provide automated control. The factory configuration uses an RS-232 digital data link (tethered or wireless) between the console processor and the onboard control processor. Analog control actions at the console are converted into digital signals and packaged and sent to the robot where they are decoded and converted back into analog signals to control the various motors on board. This design configuration permits relatively easy addition of computing power to integrate the added functions. As illustrated in Figure 2, the additional computing power is incorporated into the robot system by means of insertion into the RS-232 link.

The computing power added to the system is incorporated into a computer board cage (VME) in the form of two cards each containing a Motorola M68040 central processor unit (CPU) with associated memory and other necessary data processing devices. The cage is mounted on a custom-designed plate which attaches to the robot at the base of the pantilt camera tower is such a was that there is no permanent alteration to the configuration of the robot. This is desirable because the unit needs to be usable as a telerobot to perform tasks in contaminated areas which might arise during the course of this project. Therefore, one of the important goals of the CRADA is to be able to recover the original factory configuration of the robot, and to add the needed equipment in such a way that no permanent alterations are done which would, for example, reduce the contamination resistance of the unit.

One of the two added processors handles the incoming signals from the sensors and encoders aboard the robot. These data are processed through an analog-to-digital (AD) signal converter prior to being sent to the first processor. This processor interprets and stores the incoming data, updating the data tables with new sensor and encoder information as required. The second CPU serves as a monitor of the control signals generated by the operator and sent along the RS-232 link. This unique arrangement permits this processor to either pass the control signals along unmodified or to alter them so as to modify the commands before they reach the control CPU in the robot. When the monitor CPU provides no signal modification, the robot operates exactly as the factory delivered it, in keeping with the CRADA goal of preserving the original factory specifications as a fall-back position.

\section{Functioning of the enhanced control system}

When the added control CPU functions to alter the control signals, it serves to move the robot from a totally teleoperated mobile robot in the direction of autonomy. Figure 3 depicts the now widely accepted situation in robotics in which high degrees of autonomy are attainable only in relatively simple tasks (the area under and to the left of the curve in Figure 3). The arrow pointing to the shaded oval in the upper right indicates the direction in which we are moving with the added computing power on the Andros. As more and more task components are automated, the robot becomes more fully autonomous. With the flexibility of the present 


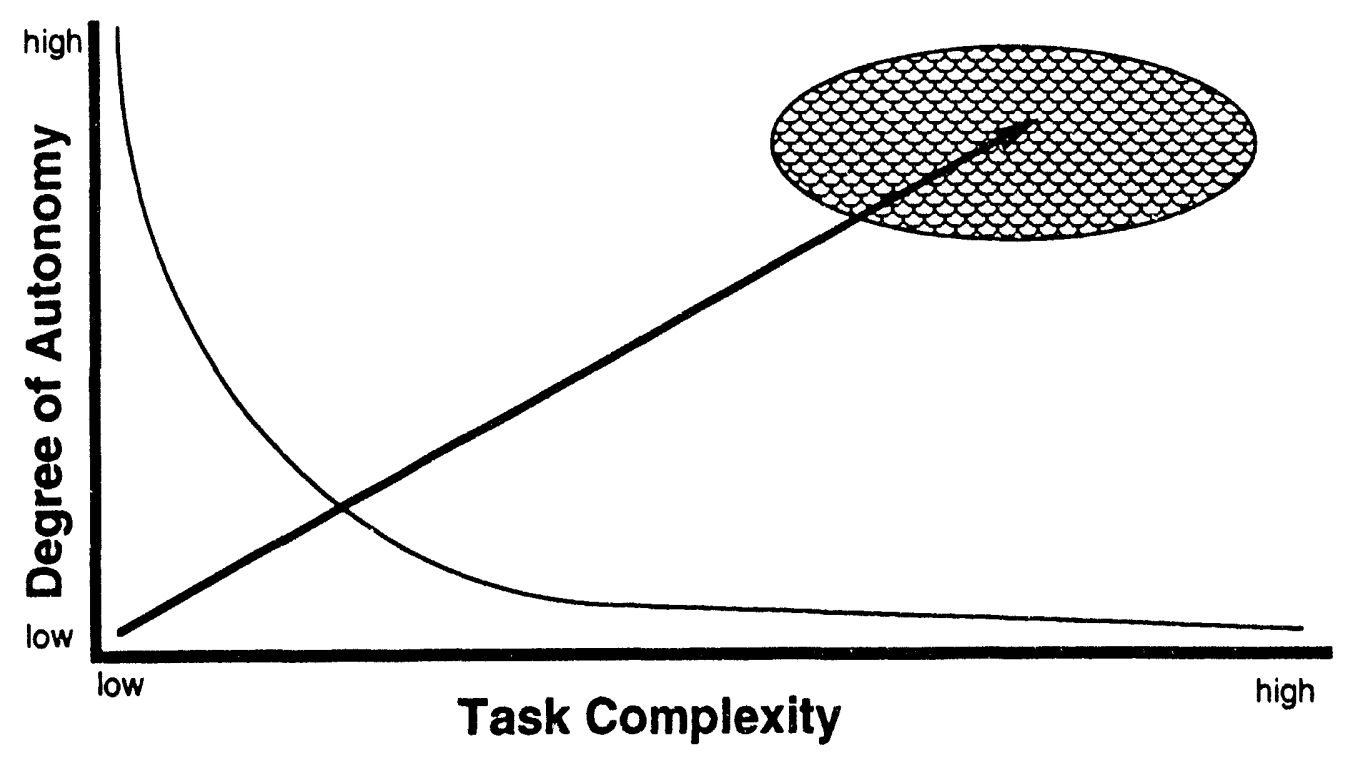

Figure 3. Diagram relating task complexity with degree of autonomy obtainable by most present-day robotic systems. The upper right oval represents the deisrable goal of high autonomy for very complex tasks.

system, different degrees of autonomy can be achieved as appropriate in different task environments.

Certain of the automated functions are planned to be permanent, while others may be invoked at some times and not at others. Many of the permanent functions fall into a class which can be designated as safety functions, and represent functions toward the lower left of the arrow in Figure 3. For example, the original robot is able to contact the pan/tilt camera tower with the manipulator arm, and it is the operator's responsibility to prevent this from occurring. With the enhanced control system in place, a software-derived envelope has been created around the camera tower, thus precluding accidental contact by the arm. Similarly, a variety of "illegal" configurations and poses can be defined which will protect both the robot and the environment from undesirable or dangerous situations. In this capacity, the CPU which monitors the control inputs simply changes the control commands to prevent the undesirable configuration from arising. This includes stopping the robot if it attempts to navigate a slope which is too steep in either pitch or roll, or if it is about to collide with an obstacle about which the operator is unaware.

Additional intelligent or automated capabilities serve to move the system toward the upper right along the arrow in Figure 3. At the simpler levels, these functions might include automated obstacle negotiation, manipulator or end effector tasks, and path planning. For example, a variety of repetitive manipulator tasks such as valve turning might be automated. In this case, the operator would position the robot so it could perform the valve closing, and the additional onboard CPU would assume the responsibility for actually closing the valve. At more complex levels of task automation (farther up and to the right in Figure 3), greater degrees of machine autonomy become involved, as more complex tasks are performed without operator intervention. This is one of the purposes of designing the enhanced operator interface for the Andros robot, and represents the type of new interface which will be fit to both existing and new examples of the robot line.

\section{Euture research on operator-machine synergy}

In addition to serving as the testbed for developing the enhanced interface just discussed, this prototype system provides the opportunity to experiment with the advantages and disadvantages of varying degrees of task automation. These issues are of current interest in both aircraft cockpit automation and in the new designs of inherently safe nuclear reacior design (Spelt, 1993). Research in these areas indicates that operator boredom and takeover transients, when operator action is required, are a source of increased human error in highly automated systems.

Certain of the automated functions are planned to be permanent, while others may be invoked at some times and not at others. Many of the permanent functions fall into a class which can be designated as safety functions, and represent functions toward the lower left of the arrow in Figure 3. For example, the original robot is able to contact the pan/tilt camera tower with the manipulator arm, and it is the operator's responsibility to prevent this from occurring. With the enhanced control system in place, a software-derived envelope has been created around the camera tower, thus precluding accidental contact by the arm. Similarly, a variety of "illegal" configurations and poses can be defined which will protect both the robot and the environment from undesirable or dangerous situations. In this capacity, the CPU which monitors the control inputs simply changes the control commands to prevent the undesirable configuration from arising. This includes stopping the robot if it attempts to navigate a slope which is too steep in either pitch or roll, or it it is about to collide with an obstacle about which the operator is unaware.

Additional intelligent or automated capabilities serve to move the system toward the upper right along the arrow 
in Figure 3. At the simpler levels, thase functions might include automated obstacle negotiation, manipulator or end effector tasks, and path planning. For example, a variety of repetitive manipulator lasks such as valve turning might be automated. In this case, the operator would position the robot so it could perform the valve closing, and the additiona! onboard CPU would assume the responsibility for actually closing the vaive. At more complex levels of task automation (farther up and to the right in Figure 3), greater degrees of machine autonomy become involved, as more complex tasks are performed without operator intervention. This is one of the purposes of designing the enhanced operator interface for the Andros robot, and represents the type of new interface which will be fit to both existing and new examples of the robot line.

\section{Euture research on epecator-machine synergy}

In addition to serving as the testbed for developing the enhanced interface just discussed, this prototype system provides the opportunity to experiment with the advantages and disadvantages of varying degrees of task automation. These issues are of current interest in both aircraft cockpit automation and in the new designs of inherently safe nuclear reactor design (Spelt, 1993). Research in these areas indicates that cperator boredom and takeover transients, when operator action is required, are a source of increased human error in highly automated systems.
Ultimately, this system has the capability to perform complex tasks autonomously, using sensor-based feedback from the environmerit. As a result, this system will serve as a research vehicle for research into the manner in which automated lask components can be seamlessly integrated with operator-performed components to yield a system which is capable of functioning in hazardous environments in a way which is both safer and more efficient than can be done urider full teleoperation. Neither the manner nor the degree of task automation are intuitively obvious to observers of this process. Systematic research is required, in a variety of situations, io explore the most effective ways of capitalizing on the capabilities of both the human operator and the inielligent robot.

Andros robots are presently used by numerous electric utilities to perform tasks in reactors where substantial exposure to radiation exists. They are also used by the armed forces, as well as numerous law enforcement agencies. The automation of task components, as well as the video graphics display of the robot's position in the environment, will enhance all tasks performed by these users, as well as enabling pertormance in terrain where the robots cannot presently perform due to lack of knowledge about, for instance, the degree of tilt of the robot. Enhanced performance of a successful industrial mobile robot leads to increased safety and efficiency of performance in hazardous environments. The addition of these capabilities will greatly enhance the utility of the robot, as well as its marketability.

\section{REFERENCE}

Spelt, P. F. Role of the operator in nuclear power plants as determined from a survey of the North American nuclear community. Proceedings of the American Nuclear Society Topical Meeting, Oak Ridge, TN, April 10-14, 1993, pp 120-127. 

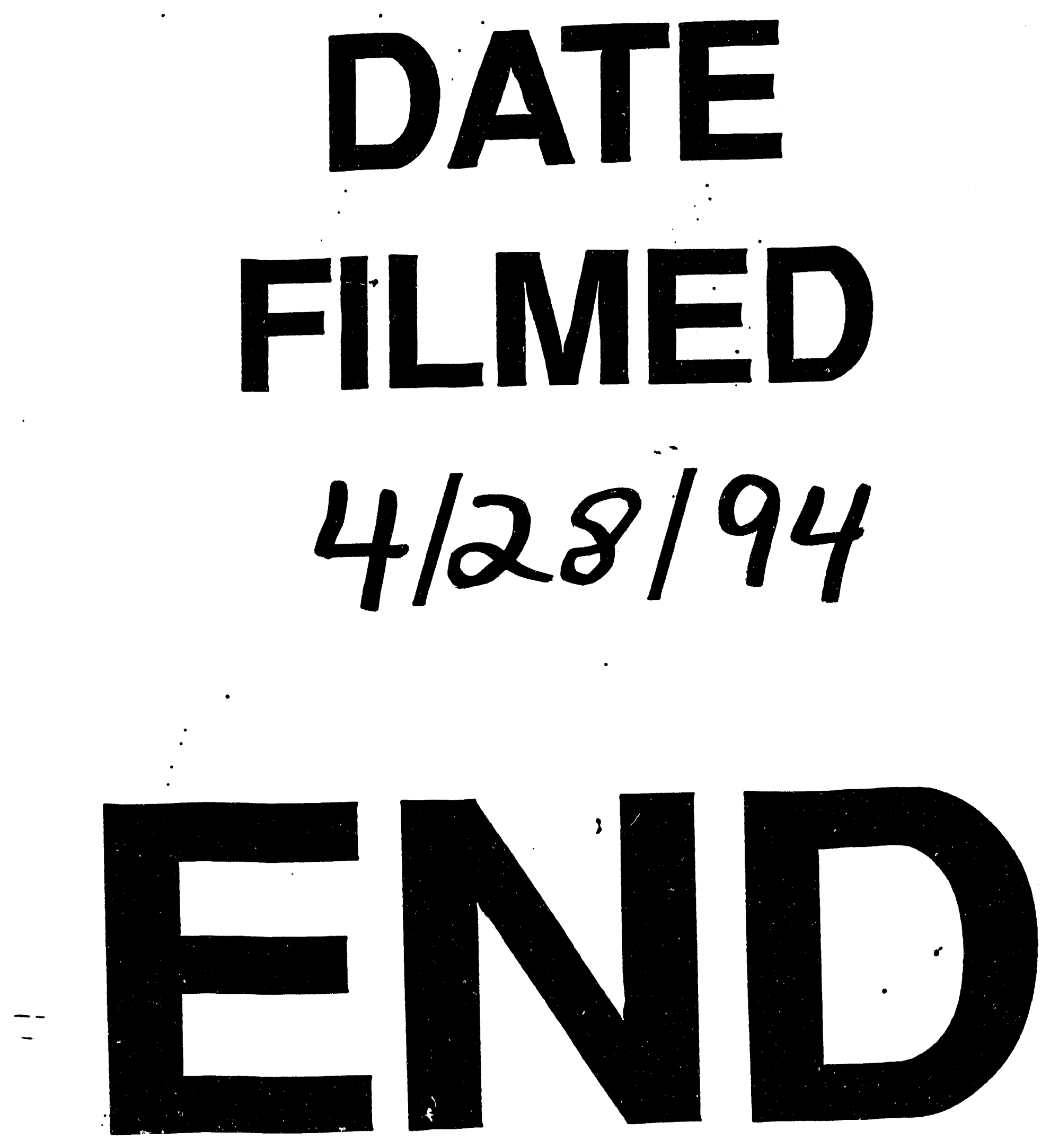
